\title{
Algorithms for Disconnected Diagrams in Lattice QCD
}

\section{Arjun Singh Gambhir*}

Department of Physics, The College of William and Mary, Williamsburg, VA, 23185, U.S.A.

Thomas Jefferson National Accelerator Facility, Newport News, VA 23606, U. S. A.

E-mail: asgambhir@email.wm.edu

\section{Andreas Stathopoulos}

Department of Computer Science, The College of William and Mary, Williamsburg, VA, 23185,

U.S.A.

E-mail: andreas@CS.wm.edu

\section{Kostas Orginos}

Department of Physics, The College of William and Mary, Williamsburg, VA, 23185, U.S.A.

Thomas Jefferson National Accelerator Facility, Newport News, VA 23606, U. S. A.

E-mail: kostasejlab.org

\section{Boram Yoon}

Los Alamos National Laboratory, Los Alamos, NM, 87545, U.S.A.

E-mail: boramelanl.gov

\section{Rajan Gupta}

Los Alamos National Laboratory, Los Alamos, NM, 87545, U.S.A.

E-mail: rgelanl.gov

\section{Sergey Syritsyn}

Department of Physics and Astronomy, Stony Brook University, Stony Brook, NY 11794, U.S.A.

E-mail: sergey.syritsynestonybrook. edu

Computing disconnected diagrams in Lattice QCD (operator insertion in a quark loop) entails the computationally demanding problem of taking the trace of the all to all quark propagator. We first outline the basic algorithm used to compute a quark loop as well as improvements to this method. Then, we motivate and introduce an algorithm based on the synergy between hierarchical probing and singular value deflation. We present results for the chiral condensate using a $2+1$-flavor clover ensemble and compare estimates of the nucleon charges with the basic algorithm.

34th annual International Symposium on Lattice Field Theory

24-30 July 2016

University of Southampton, UK

\footnotetext{
* Speaker.
} 


\section{Introduction}

Lattice QCD calculations of hadron structure observables such as form factors require computing "disconnected diagrams". These are diagrams in which a quark loop with operator insertion is coupled to the nucleon correlator via gluons only. Such diagrams are required for isoscalar and strange form factors of the nucleon.

The computation of the quark loop requires the trace of the inverse of the Dirac operator over spin, color, and spatial dimensions. An exact computation of this trace would be proportional to the square of the lattice volume, instead, stochastic methods are employed. The basic technique is a Monte Carlo (MC) averaging over matrix quadratures: the Hutchinson trace [10, 6].

$$
t\left(A^{-1}\right) \approx \frac{1}{s} \sum_{j=1}^{s} z_{j}^{\dagger} A^{-1} z_{j}
$$

where the $z_{j}$ are random noise vectors with elements that satisfy $E\left(z_{j}(k) z_{j}\left(k^{\prime}\right)\right)=\delta_{k k^{\prime}}$. In particular, for the case of $\mathbb{Z}_{4}$ noise ( $\pm 1, \pm i$ uniformly distributed elements), the variance of this estimator is

$$
\operatorname{Var}\left(\operatorname{Tr}\left(A^{-1}\right)\right)=\left(\left\|A^{-1}\right\|_{F}^{2}-\sum_{i=1}^{N}\left|A_{i, i}^{-1}\right|^{2}\right)=\left.\sum_{i \neq j}^{N} \sum_{i, j}^{-1}\right|^{2} .
$$

From equation 1.2, it is obvious that large off diagonal elements slow down the converge of the MC estimator. Many improvements to this basic estimator have been proposed and implemented in the Lattice community. Recent advances include dilution and eigenvalue deflation $[13,7,2]$.

\section{Hierarchical Probing and Deflation}

It is well known that elements of $A^{-1}$ between two points decay as a function of geometric distance on the lattice. Therefore, an improved estimator with smaller variance than that shown in eq. 1.2 should take advantage of this feature and remove or reduce the contributions from the largest off diagonal matrix elements. One possible idea it to use probing instead of MC with large numbers of $z_{j}$ [17]. This is accomplished by graph coloring the sparsity pattern produced by the Dirac matrix to generate special vectors that expose the trace. Probing removes local matrix elements up to a specified distance, thereby eliminating the largest components of error. We refer to this algorithm as classical probing $(\mathrm{CP})$.

A drawback of $\mathrm{CP}$ is that probing vectors for a particular coloring distance are not reusable at higher distances. A solution to this is to first generate $m$ probing vectors based on known structure, such as spin, color, or time. Then, $s$ random vectors are created that follow the pattern produced by each probing vector, resulting in $m \times s$ total vectors. This method is called dilution [3,12].

Hierarchical Probing (HP) expands upon CP and dilution by probing in a nested way so that the vectors at a particular probing distance are reused in all higher coloring distances. The HP basis is generated by permutations of the Hadamard matrix for lattices with purely even powers. HP annihilates the largest off diagonal elements of $A^{-1}$ in an incremental way, and has been shown to give an order of magnitude improvement over random noise for strange quark contributions [14].

Permutations of the Hadamard matrix produce a deterministic algorithm, however the estimator can be made unbiased by performing an element-wise product with a random $\mathbb{Z}_{4}$ vector. 
Therefore, HP may be viewed as an algorithm that generates a nested probing basis from any starting vector.

For light quark masses, $A^{-1}$ becomes dominated by low lying eigenvectors and the off-diagonal elements decay slowly. We, therefore, supplement HP with deflation, removal of low modes from $A$ via a projection, to recover the strong decay structure, i.e., we calculate the trace of the deflated matrix with HP and correct it by adding to it the exact low mode contribution. In our implementation, we deflate $A$ using the singular value decomposition (SVD) instead of the typical eigendecomposition. The effects of SVD deflation are studied theoretically and numerically in [9]. The improved deflated HP estimator is given by

$$
\operatorname{Tr}\left(A^{-1}\right) \approx \frac{1}{s} \sum_{j=1}^{s}\left(z_{j}^{\dagger} A^{-1} z_{j}-z_{j}^{\dagger} V \Sigma^{-1} U^{\dagger} z_{j}\right)+\operatorname{Tr}\left(V \Sigma^{-1} U^{\dagger}\right),
$$

where $U, \Sigma$, and $V$ are the low lying singular triplets of $A$.

In order for the total algorithm to be cost-effective, deflation must be extremely efficient. Possible algorithms for determining the low lying subspace are Lanzcos or eigCG [16]. We chose instead the PReconditioned Iterative MultiMethod Eigensolver (PRIMME) [15] library, accelerated with a multigrid preconditioner [1], to solve for the eigenpairs $\Lambda, V$ of $A^{\dagger} A$. This allowed us to efficiently generate 1,000 lowest singular triplets of $A$. We stress the importance and computational challenge of this problem due to the high density of the low lying spectrum of the Dirac matrix. Details of our procedure can be found in [9].

With the low mode space calculated, the "Deflated Trace Estimation Algorithm" is as follows:

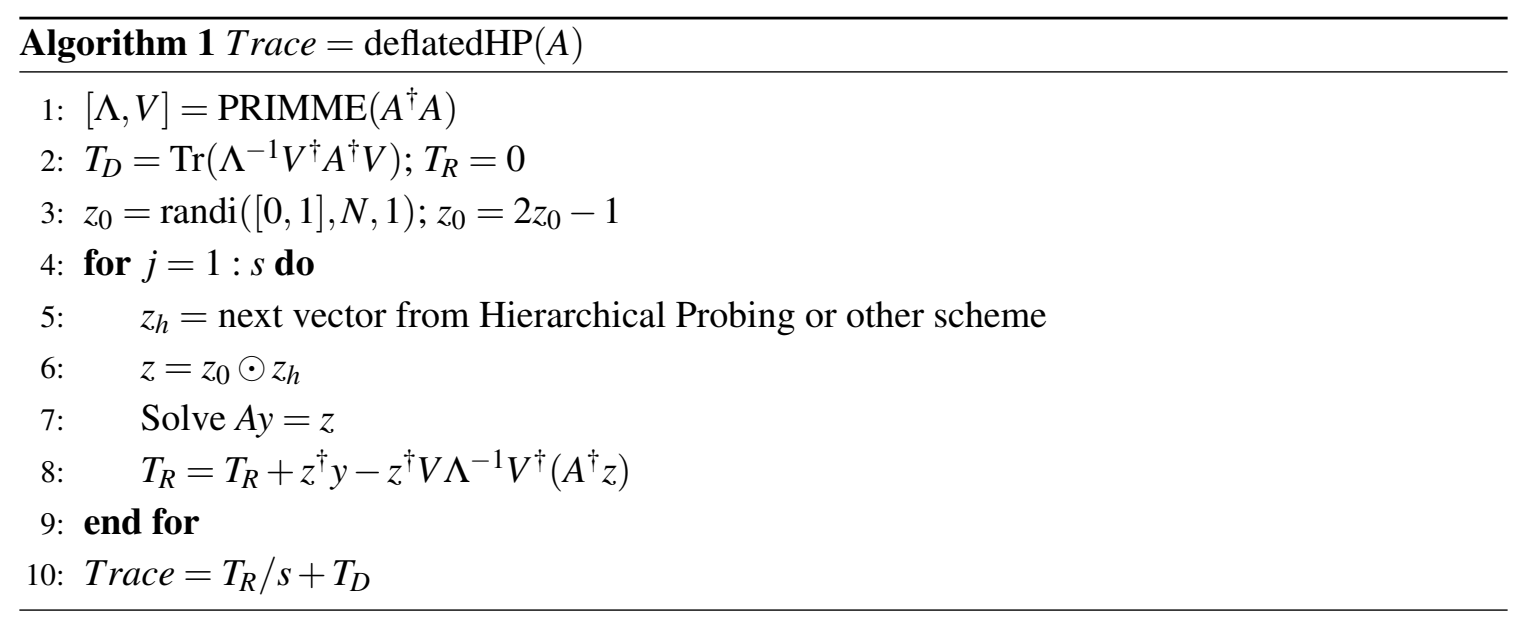

\section{Numerical Experiments}

\subsection{Variance and Speedup Tests}

We computed $\operatorname{Tr} A^{-1}$ to estimate the variance reduction and speedup of the combined, deflated HP, algorithm. These tests were performed on $32^{3} \times 642+1$-flavor Clover-on-Clover fermion lattices with spacing $a=0.0081 \mathrm{fm}$ and pion mass $m_{\pi}=312 \mathrm{MeV}$.

Figure 1a, shows that deflation reduces variance of HP by a factor of 20 at the third color closing point. The speedup in Fig. $1 \mathrm{~b}$ is given by $R_{S}=\frac{V_{\text {stoc }}}{V_{h p}(s) \times s}$ where $V_{\text {stoc }}$ is the variance of the 


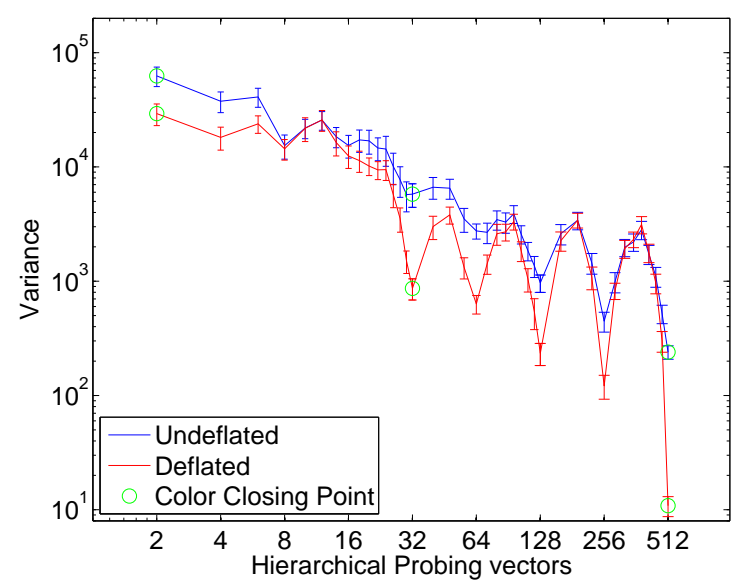

(a) Variance of $\operatorname{Tr}\left(A^{-1}\right)$ Variance

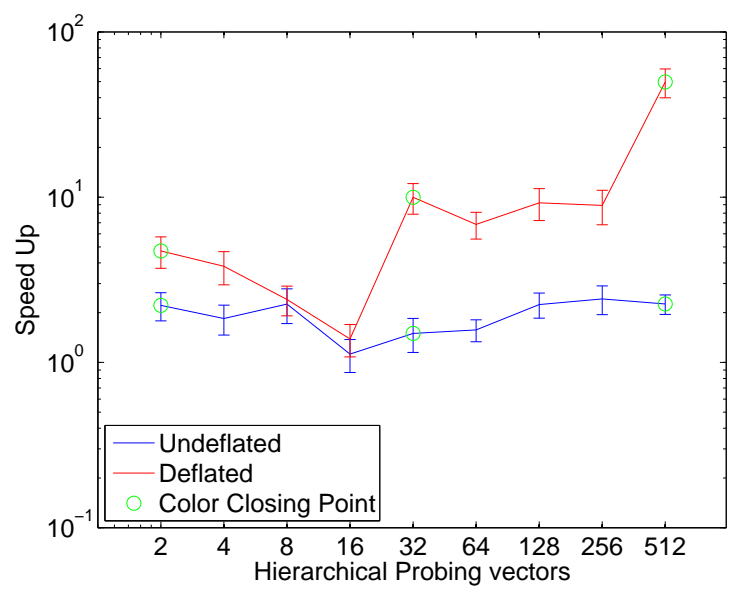

(b) Speedup of $\operatorname{Tr}\left(A^{-1}\right)$ Variance

Figure 1: The left plot shows the variance of the deflated HP trace estimator. Speedup in comparison to pure $\mathbb{Z}_{4}$ noise is shown on the right. The points 2,32 , and 512 are the color closing points of the nested basis and give the highest variance reduction, and conversely the best speedup. The errors on both figures were computed with jackknife resampling over $40 \mathbb{Z}_{4}$ noise vectors.

purely stochastic MC estimator, and $V_{h p}(s)$ is the variance of deflated HP. The factor of $s$ in the denominator normalizes against pure noise since error from the MC estimator falls off as $\sqrt{\frac{V_{s t o c}}{s}}$. Fig. $1 \mathrm{~b}$ shows that at light quark masses HP alone yields only a factor of 2-3 speedup over the basic method (blue spline). Augmented with deflation, HP gives a factor of 60 speedup at the third color closing point.

\subsection{Synergy between Deflation and HP}

To understand why these two methods, deflation and HP, work well together, we sample 10 randomly selected rows $i$ of the Dirac matrix used above, $A_{i, j}^{-1}$, as a function of their Manhattan distance $M$ along the lattice $(j)$. We exhibit results for four cases in Fig. 2: an MC estimator, deflation alone, HP alone, and deflation combined with HP. Data in Fig. 2 show that HP reduces the off-diagonal elements of $A^{-1}$ at a small Manhattan distance faster (blue squares lie below green circles and red crosses at $M \sim 4$ ). From $M=10$ to $M=50$, deflation is more effective (red crosses lie below green circles and blue squares). Together, these techniques yield a highly efficient trace estimation algorithm (black plus symbol).

\subsection{Nucleon Charges}

The effectiveness of deflated HP in computing disconnected contributions to nucleon charges is shown in Figs. 3a, 3b, 3c, and 3d. The source-sink separation in the 3-point function was $t_{\text {sep }}=10$ and the loop insertion is halfway at $\tau=5$. The two-point correlation functions in this calculation were computed using the all-mode-averaging method [5] with 96 low-precision and 3 high-precision measurements. Deflated HP was compared to an MC run using $\mathbb{Z}_{2}$ noise vectors. The $\mathrm{MC}$ run used $512 Z_{2}$ noise vectors improved with the hopping parameter expansion $[8,11]$ and the truncated solver method [4]. 


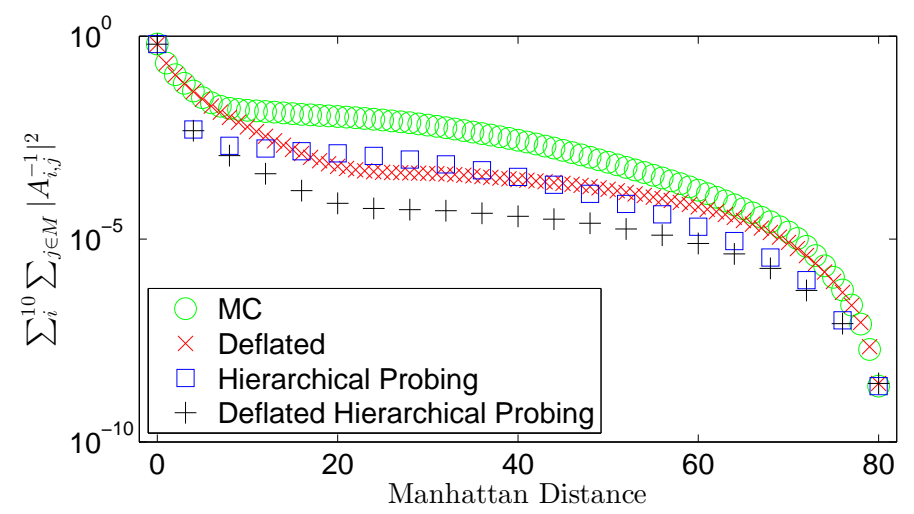

Figure 2: Deflation alone includes the full space of 1,000 singular triplets. HP alone is the application of 32 hadamard vectors. Deflated HP is the combination of both methods.

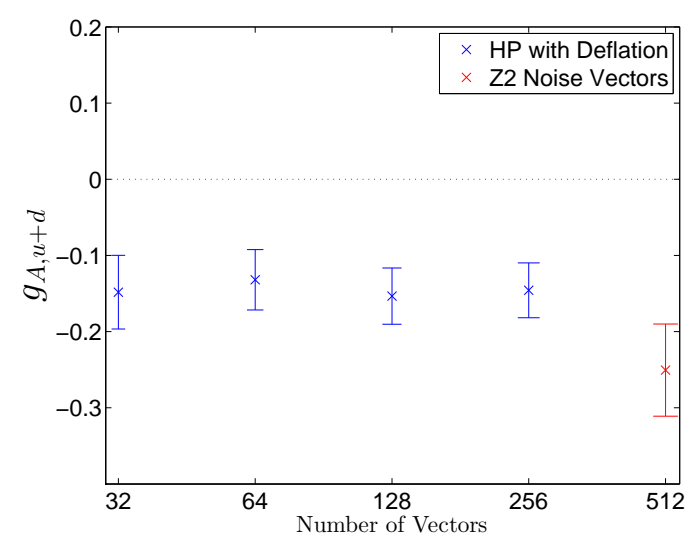

(a) $g_{A} 100 \mathrm{cfgs}$

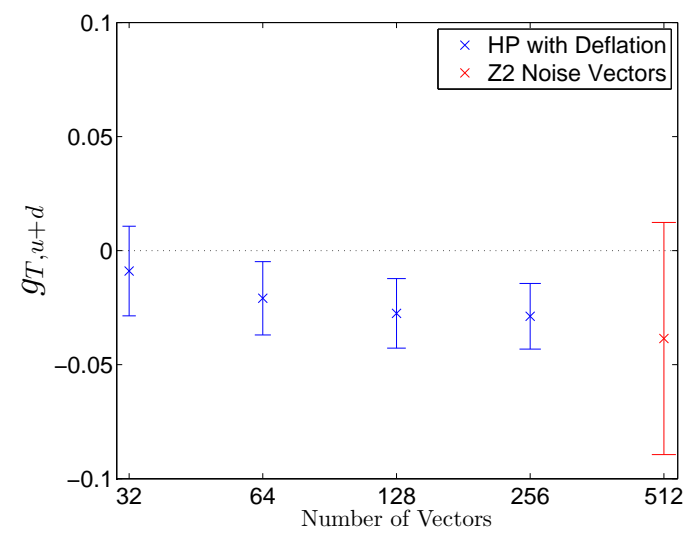

(c) $g_{T} 100 \mathrm{cfgs}$

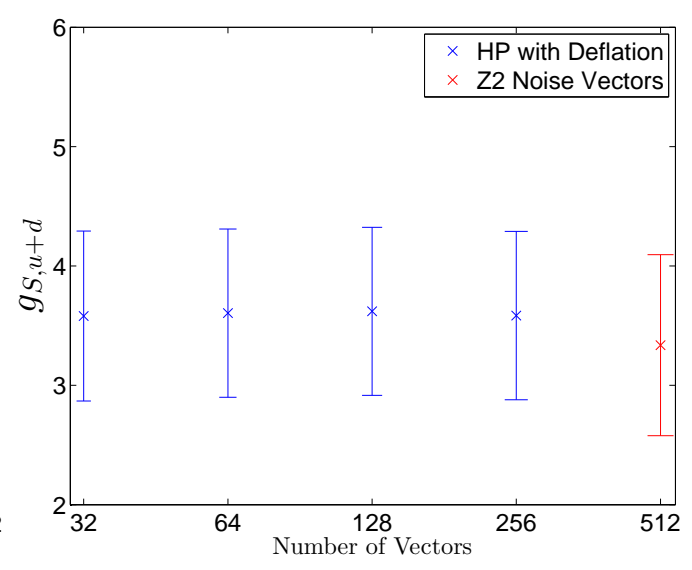

(b) $g_{S} 100 \mathrm{cfgs}$

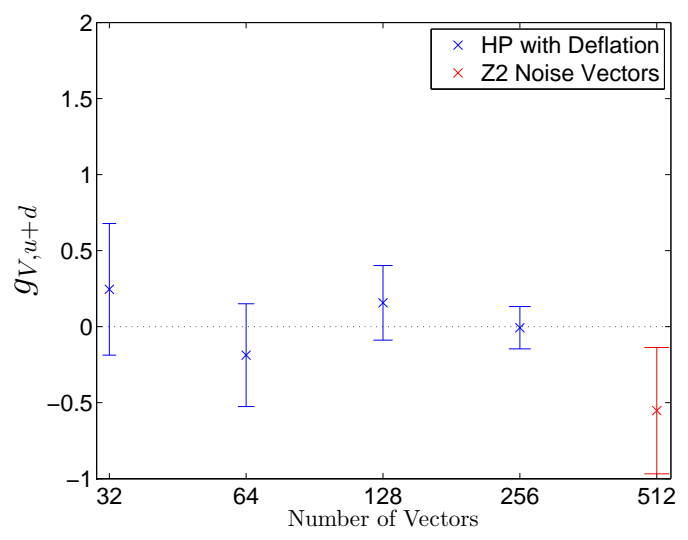

(d) $g_{V} 100 \mathrm{cfgs}$

Figure 3: $g A g S, g T$, and $g V$ computed with 32, 64, 128, and $256 \mathrm{HP}$ vectors and 500 deflation vectors, compared to the MC estimate with $512 \mathbb{Z}_{2}$ vectors.

The data in Figs. 3a, 3b, 3c, and 3d show that deflated HP with $32 \mathrm{HP}$ vectors performed better than $512 Z_{2}$ noise vectors. The cost of generating the 500 deflation vectors was approximately $20 \%$ 
of the cost of the 256 probing vectors. This can be computed from the timings in Re. [9]. For the case of $g_{A}, g_{T}$, and especially $g_{V}$, substantial error reduction was observed with the Deflation HP method. For the scalar charge, $g_{S}$, fluctuations from the ensemble average are large and contribute more significantly to the error. Thus a highly accurate trace per configuration is not beneficial.

\section{Conclusions and Outlook}

We emphasize the synergy of the two algorithms: Hierarchical Probing (HP) and Deflation. Although HP's effectiveness decreases at light quark masses, substantial variance reduction is achieved when augmented with deflation. Disconnected contributions to the three nucleon charges $g_{A, T, V}$ benefit from our more efficient trace estimator, while little improvement is observed for $g_{S}$. We are currently extending our analysis to isoscalar vector and axial vector form factors: $G_{E}^{s}\left(Q^{2}\right)$, $G_{M}^{s}\left(Q^{2}\right), G_{A}^{s}\left(Q^{2}\right)$, and $G_{S}^{s}\left(Q^{2}\right)$.

\section{Acknowledgments}

This work has been supported by NSF under grants No. CCF 1218349 and ACI SI2-SSE 1440700, and by DOE under a grant No. DE-FC02-12ER41890. KO and AG have been supported by the U.S. Department of Energy through Grant Number DE- FG02-04ER41302. KO has been supported through contract Number DE-AC05-06OR23177 under which JSA operates the Thomas Jefferson National Accelerator Facility. AG has been supported by the U.S. Department of Energy, Office of Science, Office of Workforce Development for Teachers and Scientists, Office of Science Graduate Student Research (SCGSR) program. The SCGSR program is administered by the Oak Ridge Institute for Science and Education for the DOE under contract number DE-AC0506OR23100. This research used resources of the National Energy Research Scientific Computing Center, a DOE Office of Science User Facility supported by the Office of Science of the U.S. Department of Energy under Contract No. DE-AC02-05CH11231. Additionally, this research used resources of the Oak Ridge Leadership Computing Facility at the Oak Ridge National Laboratory, which is supported by the Office of Science of the U.S. Department of Energy under Contract No. DE-AC05-00OR22725. The work of R.G. and B.Y. is supported by the U.S. Department of Energy, Office of Science, Office of High Energy Physics under contract number DE-KA-1401020 and the LANL LDRD program.

\section{References}

[1] R. Babich, J. Brannick, R. C. Brower, M. A. Clark, T. A. Manteuffel, S. F. McCormick, J. C. Osborn, and C. Rebbi. Adaptive multigrid algorithm for the lattice Wilson-Dirac operator. Phys. Rev. Lett., 105:201602, 2010.

[2] R. Babich, R. Brower, M. Clark, G. Fleming, J. Osborn, and C. Rebbi. Strange quark contribution to nucleon form factors. PoS, LAT2007:139, 2007.

[3] R. Babich, R. Brower, M. Clark, G. Fleming, J. Osborn, C. Rebbi, and D. Schaich. Exploring strange nucleon form factors on the lattice. 4 May 2011. 
[4] G. S. Bali, S. Collins, and A. Schaefer. Effective noise reduction techniques for disconnected loops in Lattice QCD. (arXiv hep-lat 0910.3970v2), 2010.

[5] Thomas Blum, Taku Izubuchi, and Eigo Shintani. New class of variance-reduction techniques using lattice symmetries. Phys. Rev., D88(9):094503, 2013.

[6] Shao-Jing Dong and Keh-Fei Liu. Stochastic estimation with Z(2) noise. Phys. Lett., B328:130-136, 1994.

[7] J. Foley., K. J. Juge, A. O’Cais, M. Peardon, S. Ryan, and J.-I. Skullerud. Practical all-to-all propagators for lattice qcd. Comput. Phys. Commun., 172:145-162, 2005.

[8] M. Foster and Christopher Michael. Quark mass dependence of hadron masses from lattice QCD. Phys. Rev., D59:074503, 1999.

[9] Arjun Singh Gambhir, Andreas Stathopoulos, and Kostas Orginos. Deflation as a Method of Variance Reduction for Estimating the Trace of a Matrix Inverse. 2016.

[10] M. F. Hutchinson. A stochastic estimator of the trace of the influence matrix for Laplacian smoothing splines. J. Commun. Statist. Simula., 19(2):433-450, 1990.

[11] Christopher Michael, M. S. Foster, and C. McNeile. Flavor singlet pseudoscalar and scalar mesons. Nucl. Phys. Proc. Suppl., 83:185-187, 2000.

[12] C. Morningstar, J. Bulava, J. Foley, K.J. Juge, D. Lenkner, M. Peardon, and C.H. Wong1. Improved stochastic estimation of quark propagation with Laplacian Heaviside smearing in lattice QCD. Phys. Rev. D, 83(114505), 2011.

[13] H. Neff, N. Eicker, T. Lippert, John W. Negele, and K. Schilling. On the low fermionic eigenmode dominance in QCD on the lattice. Phys. Rev., D64:114509, 2001.

[14] Andreas Stathopoulos, Jesse Laeuchli, and Kostas Orginos. Hierarchical probing for estimating the trace of the matrix inverse on toroidal lattices. 2013.

[15] Andreas Stathopoulos and James R. McCombs. Primme: Preconditioned iterative multimethod eigensolver: Methods and software description, 2006.

[16] Andreas Stathopoulos and Kostas Orginos. Computing and deflating eigenvalues while solving multiple right hand side linear systems in quantum chromodynamics. SIAM J. Sci. Comput., 32:439-462, 2010.

[17] J. Tang and Yousef Saad. Domain-decomposition-type methods for computing the diagonal of a matrix inverse. Report UMSI 2010/114, (MSI, University of Minnesota). 